\title{
Triplet state dynamics within cyclodextrin solid complexes
}

\author{
Mónica Barra,* Christine DeVisser, Angela M. Swartz, and Christiana Zaharia \\ Department of Chemistry, University of Waterloo, Waterloo, Ontario, Canada N2L 3G1 \\ E-mail: mbarra@sciborg.uwaterloo.ca
}

\begin{abstract}
Dedicated to Professor Roberto A. Rossi on his $60^{\text {th }}$ birthday and Professor Edmundo A. Rúveda on his $70^{\text {th }}$ birthday
\end{abstract}

(received 23 May 03; accepted 09 Jul 03; published on the web 13 Jul 03)

\begin{abstract}
Time-resolved diffuse-reflectance laser-flash photolysis techniques were employed to determine the triplet state dynamics of 9,10-anthraquinone, benzil, 1,4-benzoquinone, 2,5-dimethyl-1,4benzoquinone, 9,10-phenanthrenequinone, and $\alpha$-terthienyl within $\beta$-cyclodextrin solid complexes. The $\alpha$ - and $\gamma$-cyclodextrin solid complexes of benzil and $\alpha$-terthienyl were studied likewise. The average triplet lifetime for $\alpha$-terthienyl increases as the size of the host cavity decreases, while that for benzil remains practically unchanged. In addition, the solid-state photoreduction of 1,2-dimethoxy-4-nitrobenzene triplets by 1-phenylethylamine within $\beta$-cyclodextrin solid complexes was investigated. Observed lifetimes tend to diminish as the 1-phenylethylamine-to-1,2-dimethoxy-4-nitrobenzene molar ratio increases.
\end{abstract}

Keywords: Cyclodextrins, diffuse-reflectance, lifetime distributions, solid inclusion compounds

\section{Introduction}

Cyclodextrins (CDs), cyclic oligosaccharides of six to eight $\alpha$-D-glucose units (namely $\alpha, \beta$, and $\gamma$-CD, respectively), have been widely used as hosts over the past decades, ${ }^{1}$ and continue to be the subjects of current interest both in basic and applied research. ${ }^{2}$ Despite numerous studies on photophysical and photochemical properties of organic guest species complexed with cyclodextrins in the solid state, ${ }^{3}$ there is only a handful of publications on the kinetics of photoinduced transient species within cyclodextrin solid complexes. ${ }^{4-13}$ The work reported here focuses on the application of time-resolved diffuse-reflectance laser-flash photolysis techniques to characterize the triplet state dynamics of several aromatic compounds within cyclodextrin solid complexes. The aromatic guest substrates employed in this study are summarized in Chart 1; all of these substrates are known to form excited triplet states in essentially quantitative 
yield. ${ }^{14}$ In addition, the kinetics associated with the solid-state photoreduction of 1,2-dimethoxy4-nitrobenzene triplets by 1-phenylethylamine within $\beta$-CD complexes is presented.

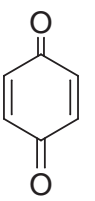

BQ<smiles>CC1=CC(=O)C(C)=CC1=O</smiles>

DMBQ<smiles>O=C(C(=O)c1ccccc1)c1ccccc1</smiles>

BZ<smiles>O=C1c2ccccc2C(=O)c2ccccc21</smiles>

$A Q$<smiles>O=C1C(=O)c2ccccc2-c2ccccc21</smiles>

$P Q$

Chart 1. Target guest species.

\section{Results and Discussion}

\section{Triplet-triplet absorption spectra}

Laser excitation (at $355 \mathrm{~nm}$ ) of any of the cyclodextrin solid complexes for the guest species shown in Chart 1, leads to the instantaneous formation (i.e., within the time response of the nanosecond laser-flash photolysis system used) of the corresponding guest excited triplet state. This in inferred by comparison of the resulting transient absorption spectra with the spectral data reported in the literature for excited triplet states in solution and in other solid systems. For instance, in the case of 1,4-benzoquinone (BQ) and 2,5-dimethyl-1,4-benzoquinone (DMBQ)/ $\beta$ CD solid complexes, transient spectra are characterized by an absorption band centered at ca. 400-410 nm (Figures 1A-B). This band agrees very well with the triplet-triplet (T-T) absorption spectrum observed in aqueous solutions, albeit slightly blue-shifted in the case of DMBQ. ${ }^{15}$ Regardless of the type of CD employed, laser excitation of benzil (BZ)/CD solid complexes leads to a transient absorption band centered at ca. $480 \mathrm{~nm}$ (Figure 1C). This band agrees excellently with the T-T absorption spectra observed in cyclohexane and acetonitrile solutions, ${ }^{16}$ as well in silicalite ${ }^{17}$ and $\beta-\mathrm{CD}^{10}$ solid matrices. Likewise, laser excitation of $\alpha$-terthienyl $(\alpha \mathbf{T}) / \mathrm{CD}$ solid complexes leads to a transient absorption band centered at ca. $470 \mathrm{~nm}$ (Figure 1D), independently of the type of CD employed. These spectra are very similar to the T-T spectra observed for $\alpha \mathbf{T}$ in organic solvents such as methanol, ${ }^{18}$ as well as in $\beta$-CD aqueous solutions. $^{19}$

The transient absorption spectra shown in Figures $1 \mathrm{~A}-\mathrm{D}$ seem to retain their shape through time, i.e., only one transient species is being detected. On the contrary, in the case of 9,10- 
anthraquinone (AQ) and 9,10-phenanthrenequinone (PQ)/ $\beta$-CD solid complexes, the spectral changes seem to indicate the presence of (at least) two absorbing species (Figures 1E-F).
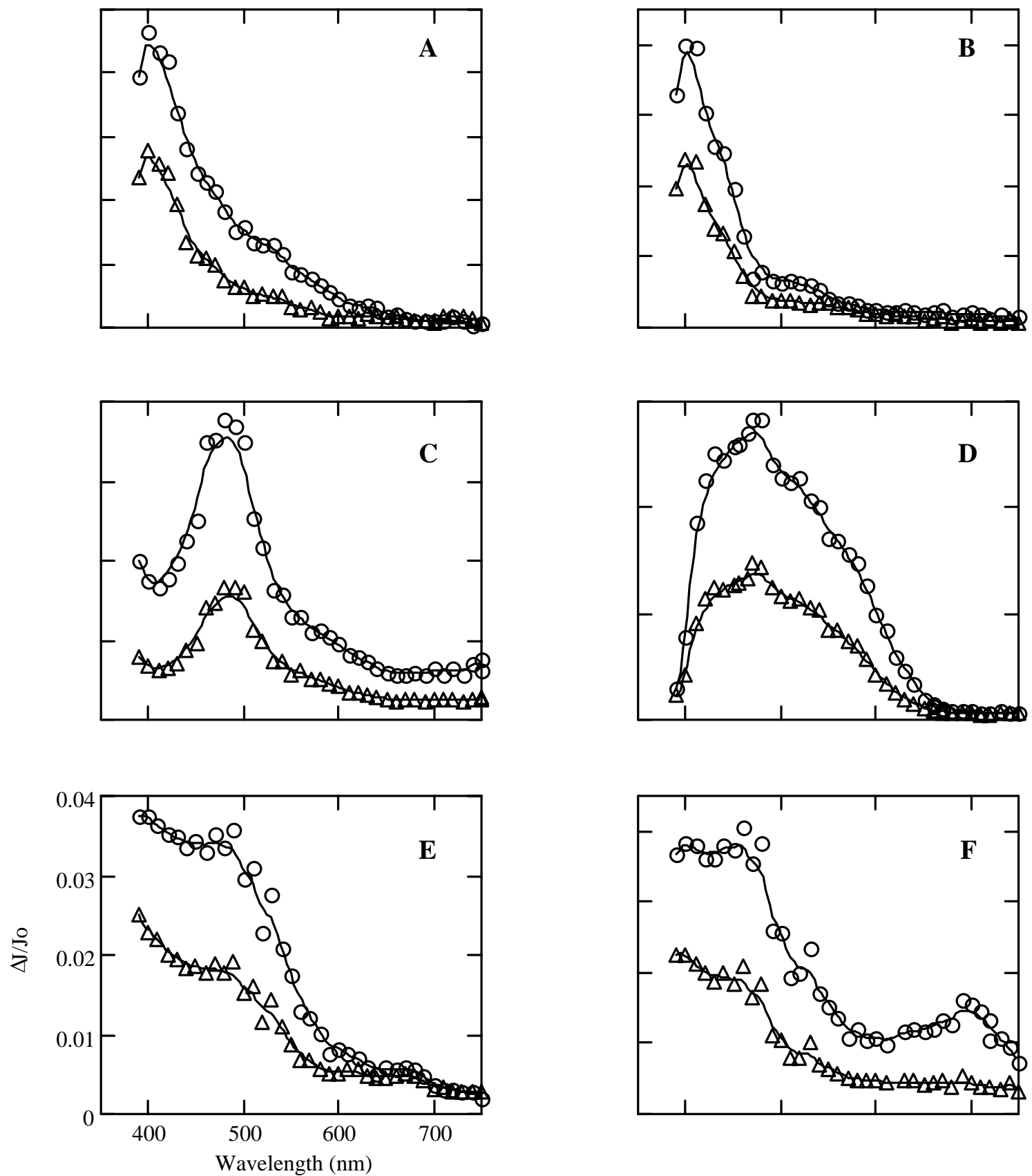

Figure 1. Transient absorption spectra for $\mathbf{B Q} / \beta$-CD (A, obtained $10 \mu \mathrm{s}(\mathrm{O})$ and $73.2 \mu \mathrm{s}(\Delta)$ after laser pulse), DMBQ $/ \beta$-CD (B, obtained $8 \mu \mathrm{s}(\mathrm{O})$ and $72.8 \mu \mathrm{s}(\Delta)$ after laser pulse), BZ/ $\beta$-CD (C, obtained $6.8 \mu \mathrm{s}(\mathrm{O})$ and $67.6 \mu \mathrm{s}(\Delta)$ after laser pulse), $\alpha \mathrm{T} / \beta$-CD (D, obtained $6.8 \mu \mathrm{s}(\mathrm{O})$ and 68.8 $\mu$ s $(\Delta)$ after laser pulse), AQ/ $\beta$-CD (E, obtained $7.2 \mu$ s $(\mathrm{O})$ and $68.4 \mu \mathrm{s}(\Delta)$ after laser pulse) and $\mathbf{P Q} / \beta$-CD (F, obtained $6.4 \mu \mathrm{s}(\mathrm{O})$ and $71.2 \mu \mathrm{s}(\Delta)$ after laser pulse). 
The ease with which triplet states of quinones abstract hydrogen from hydrogen donating reactants is very well documented. ${ }^{14 a}$ The transient spectra shown in Figures 1E-F are consistent with the presence of excited triplet states and corresponding semiquinone radicals. For instance, the transient absorption spectra of $\mathbf{P Q} / \beta$-CD solid complexes show peaks centered at ca. 390, 460 and $700 \mathrm{~nm}$. The peaks at 460 and $700 \mathrm{~nm}$ are attributed to the excited triplet state, ${ }^{20}$ while the peak at $390 \mathrm{~nm}$ is assigned to the radical (semiquinone) species. ${ }^{21}$ In the case of $\mathbf{A Q} / \beta-\mathrm{CD}$ solid complexes, analysis of the transient spectra is rather difficult as a result of the significant overlap between the T-T and radical absorptions in the 390-500 nm region. ${ }^{22}$ However, the coexistence of triplets and radicals is inferred from the fact that kinetic traces (see next section) vary with monitoring wavelength (the shorter the monitoring wavelength, the longer the lifetime). Furthermore, it should be pointed out here that photoexcited anthraquinone-substituted $\beta$-CDs have been shown to react, in the absence of a hydrogen donating solvent, by rapid intramolecular hydrogen abstraction from the cyclodextrin itself. $^{23}$

As already mentioned, in the case of $\mathbf{B Q}$ and $\mathbf{D M B Q} / \beta-\mathrm{CD}$ solid complexes the corresponding transient absorption spectra retain their shape through time. Thus, if any hydrogen abstraction is indeed taking place the corresponding semiquinone radicals $\left(\lambda_{\max } \approx 440 \mathrm{~nm}\right)^{24}$ must be decaying rapidly (i.e. within the time response of the nanosecond laser-flash photolysis system used) by back hydrogen abstraction. Likewise, in the case of BZ/CD solid complexes no evidence for benzil ketyl radicals $\left(\lambda_{\max } \approx 360 \mathrm{~nm}\right)^{25}$ formation was obtained.

\section{Triplet state dynamics}

Transient decay traces corresponding to air-equilibrated CD solid complexes were collected on different time scales. ${ }^{26}$ Typical stretched representations (i.e., reflectance change vs. $\log ($ time)) of such traces are shown in Figure 2. Representations of these types are most convenient for systems involving transient kinetics covering several orders of magnitude in time scale, as usually observed in solid systems.; multi-component (dispersed) kinetics are generally attributed to diversity in environments/inclusion sites and of conformational structures.

Several models and methods of analysis for transient kinetics in (heterogeneous) solid systems have been reported. In recent years, lifetime distribution analysis and the Albery model have been applied to analyze transient kinetics in CD solid complexes. The Albery model accounts for dispersed kinetics assuming a Gaussian distribution of the logarithm of the rate constant about some mean value, and has two adjustable parameters: a mean rate constant $(\overline{\mathrm{k}})$ and the width of the distribution $(\gamma)$. Alternatively, lifetime distribution analysis (carried out by using the exponential series method, ESM) consists of a fitting function containing up to 100 exponential terms (with fixed, logarithmically spaced lifetimes) and variable pre-exponential factors.

The results of the ESM analysis of the transient decay traces for the CD solid complexes used in this study are illustrated in Figure 3. The lifetime range for the ESM analysis was set between 10 ns and $250 \mu$ s (limits of the laser-flash photolysis system employed); however, in no 

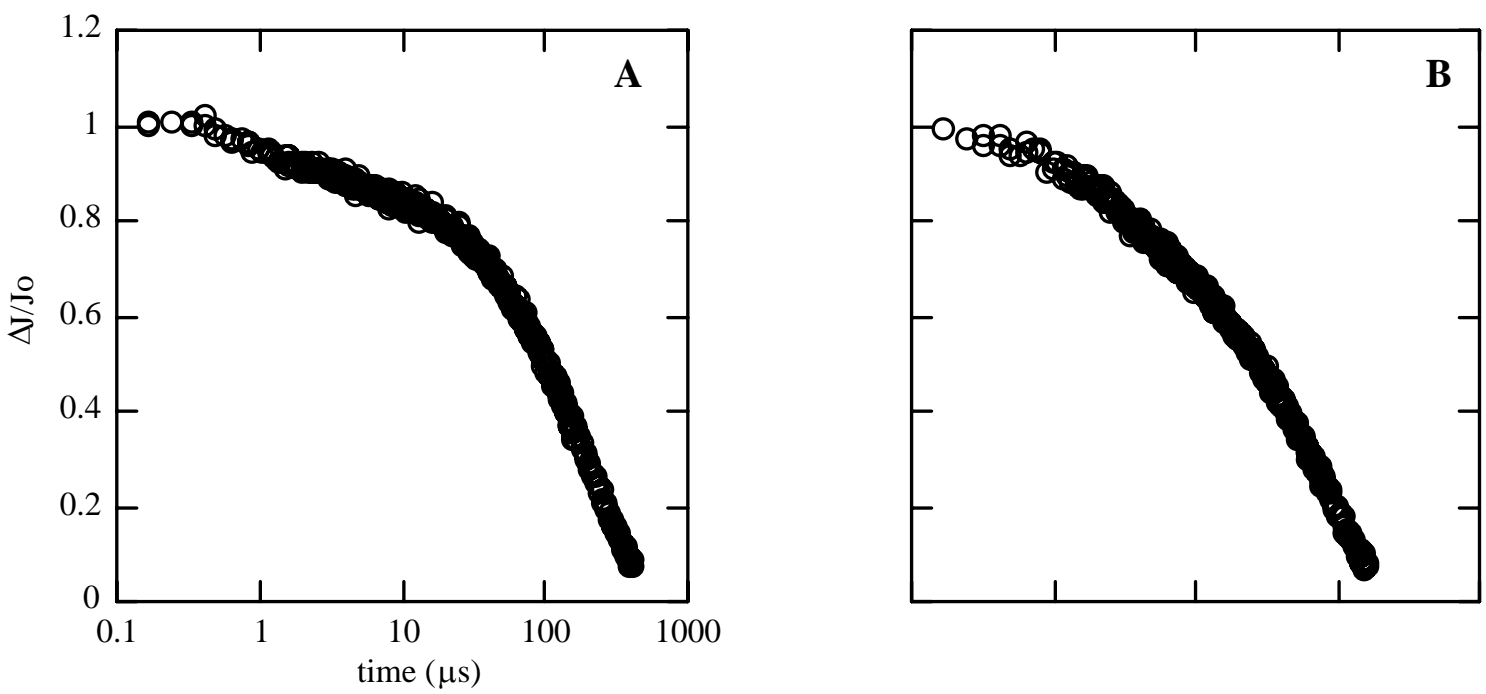

Figure 2. Normalized decay traces recorded in different time domains for: (A) $\mathbf{D M B Q} / \beta-C D$ solid complex (monitored at $420 \mathrm{~nm}$ ), and (B) $\alpha \mathbf{T} / \gamma$-CD solid complex (monitored at $470 \mathrm{~nm}$ ).

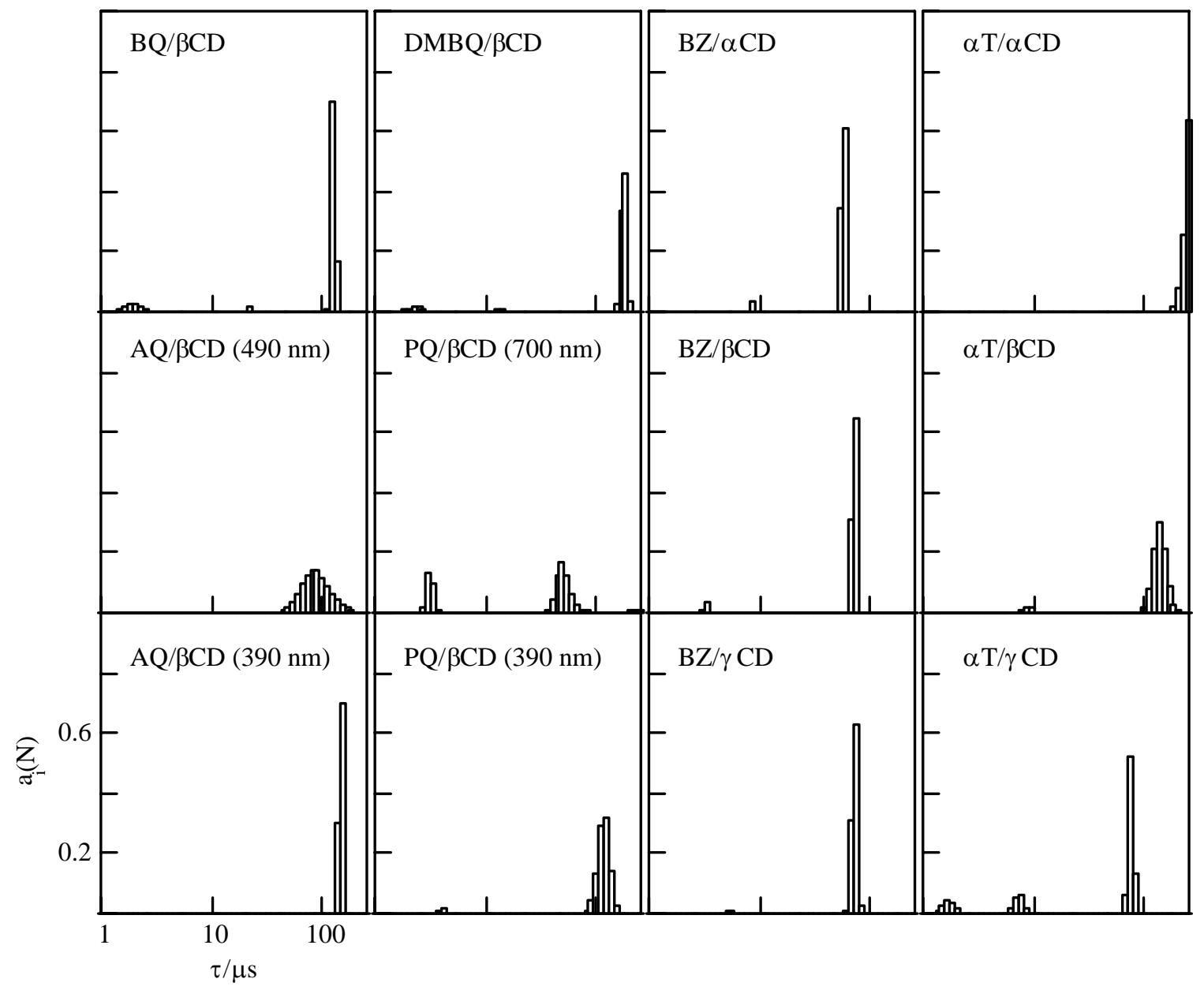

Figure 3. Lifetime distributions. 
case components of significant amplitude were obtained below $1 \mu$ s. From these ESM distributions, amplitude average lifetimes $\left(<\tau>_{\mathrm{a}}\right)$ were calculated according to eq. $(1){ }^{27}$

$$
<\tau>_{a}=\sum_{i}^{M} a_{i}(N) \times \tau_{i}
$$

where $a_{i}(N)$ represents the normalized amplitude (i.e. $\left.\Sigma a_{i}(N)=1\right)$ of the ith component, $\tau_{i}$ is its lifetime, and $\mathrm{M}$ is the number of components of the fit. The resulting $\langle\tau\rangle_{\mathrm{a}}$ values are summarized in Table 1.

Table 1. Amplitude average lifetimes $\left(<\tau>_{a}\right)$, results from the Albery model, and exponential (least-squares fitting) analysis results for air-equilibrated CD solid complexes

\begin{tabular}{lllllllll}
\hline Guest & Host & $\mathrm{H}: \mathrm{G}^{\mathrm{a}}$ & $\lambda / \mathrm{nm}^{\mathrm{b}}$ & $\left\langle\tau>_{\mathrm{a}} / \mu \mathrm{s}\right.$ & $\overline{\mathrm{k}} / 10^{-3} \mathrm{~s}^{1}$ & $\gamma$ & $\tau_{1} / \mu \mathrm{s}$ & $\tau_{2} / \mu \mathrm{s}^{\mathrm{c}}$ \\
\hline BQ & $\beta-\mathrm{CD}$ & $10: 1$ & 420 & 110 & 8.60 & 0.64 & 123 & $2(12)$ \\
DMBQ & $\beta-\mathrm{CD}$ & $10: 1$ & 420 & 152 & 6.05 & 0.65 & 173 & $2(13)$ \\
AQ & $\beta-\mathrm{CD}$ & $16: 1$ & 490 & 91 & 11.4 & 0.41 & 81 & \\
& & & 390 & 146 & 6.89 & 0.10 & 145 & \\
PQ & $\beta-\mathrm{CD}$ & $126: 1$ & 700 & 38 & 38.4 & 2.1 & 50 & $3(32)$ \\
& & & 460 & 98 & 8.47 & 0.95 & 105 & $24(17)$ \\
& & & 390 & 113 & 8.85 & 0.43 & 110 & \\
BZ & $\alpha-\mathrm{CD}$ & $383: 1$ & 490 & 56 & 18.0 & 0.30 & 55 & \\
& $\beta-\mathrm{CD}$ & $8: 1$ & 480 & 67 & 14.8 & 0.24 & 68 & \\
& $\gamma-\mathrm{CD}$ & $35: 1$ & 480 & 69 & 14.4 & 0.29 & 69 & \\
$\alpha \mathrm{T}$ & $\alpha-\mathrm{CD}$ & $2540: 1$ & 470 & $\mathrm{~d}$ & $\mathrm{~d}$ & $\mathrm{~d}$ & $\mathrm{~d}$ & \\
& $\beta-\mathrm{CD}$ & $125: 1$ & 470 & 129 & 7.65 & 0.98 & 135 & $7(6)$ \\
& $\gamma-\mathrm{CD}$ & $58: 1$ & 470 & 54 & 21.4 & 1.7 & 69 & $4(24)$ \\
\hline
\end{tabular}

${ }^{\mathrm{a}}$ Host-to-guest molar ratio. ${ }^{\mathrm{b}}$ Monitoring wavelength. ${ }^{\mathrm{c}}$ Percent contribution to total decay given in brackets. ${ }^{\mathrm{d}}$ Lifetime(s) $>250 \mu$ s (longest lifetime accessible with the laser-flash photolysis system employed).

With the exception of the ESM analysis for $\mathbf{P Q} / \beta-C D$ at $700 \mathrm{~nm}$, lifetime distributions are overall characterized by one main decay mode, which accounts for at least $80 \%$ of the decay kinetics. As illustrated in Figure 3 and summarized in Table 1, the amplitude average lifetime for $\alpha \mathbf{T}$ clearly diminishes as the size of the host cavity increases, while that for $\mathbf{B Z}$ seems to be independent of the host size, albeit a small increase (if at all significant) is observed when going from $\alpha$ - to $\beta$-CD. In the case of $\mathbf{A Q}$ and $\mathbf{P Q}$, it is clear that the amplitude average lifetimes 
diminish as the monitoring wavelength increases from $\lambda_{\max }$ of the radical (ca. $390 \mathrm{~nm}$ ) to $\lambda_{\max }$ of the triplet (> $450 \mathrm{~nm})$.

Transient decay traces were also fitted to a logarithm-normal Gaussian lifetime distribution (using the Albery model) and, based on the relatively simple pattern of the ESM distributions, to either a single or double-exponential function. As shown in Table 1 , the resulting mean rate constants $(\mathrm{k})$ and lifetimes $(\tau)$ are overall in good agreement with the corresponding amplitude average lifetimes. However, it is necessary to point out that for those samples characterized by (at least) two decay modes (e.g., PQ/ $\beta$-CD @ $700 \mathrm{~nm}$ ), fittings to the Albery model render residual plots (not shown) lacking a flat and random pattern, particularly in the initial part of the decay traces.

As indicated in Table 1, the CD solid complexes being studied are far from being stoichiometric host-guest complexes; most CD cavities are believed to be empty following loss (during drying) of diethyl ether from CD-solvent complexes that co-precipitate under the experimental conditions employed (see Experimental Section). The increase in host-to-guest molar ratio observed for $\alpha \mathbf{T}$ with decreasing host size reflects the limited space available for inclusion; this trend in host-to-guest molar ratio (and hence, in space limitations) clearly parallels the increase observed in lifetimes with decreasing host size. In the case of $\mathbf{B Z}$, the host-to-guest molar ratio increases in the order $\alpha-<\gamma-<\beta-C D$, in agreement with the fact that benzene has been shown to have maximum host-guest contact with the cavity of $\beta$-CD. ${ }^{28}$

\section{Triplet state quenching}

ESM analyses were also carried out to characterize the transient kinetics associated with the solid-state photoreduction of 1,2-dimethoxy-4-nitrobenzene (DMNB) triplets by 1-phenylethylamine (PhEA) within $\beta$-CD solid complexes.

Laser excitation of $\mathbf{D M N B} / \beta-\mathrm{CD}$ solid samples leads to the formation of DMNB excited triplet states, which are characterized by an absorption band centered at ca. $470 \mathrm{~nm}$ (Figure 4A). Upon co-inclusion of PhEA, laser excitation yields a transient absorption with a maximum at ca. $480 \mathrm{~nm}$, which undergoes a small hypsochromic shift at longer timescales (Figure 4B). These spectral changes are attributed to the formation of DMNB-H radicals (i.e., conjugate acid of DMNB radical anions), upon electron and proton transfers from PhEA to DMNB triplets within the $\beta$-CD complexes. ${ }^{13}$

Unlike the results presented in the previous section, ESM analysis corresponding to DMNB/ $\beta-C D$ and PhEA/DMNB/ $\beta-C D$ solid complexes leads to more complex lifetime distributions (Figure 4, Inset).

Solid complexes of different PhEA-to-DMNB molar ratios were prepared by changing the amount of PhEA (relative to that of DMNB and of $\beta$-CD) initially dissolved in water (see Experimental Section). As illustrated in Figure 4, lifetime distributions for PhEA/DMNB/ $\beta$-CD solid samples are much broader than those corresponding to $\mathbf{D M N B} / \beta-C D$ complexes. Due to the significant overlap between the triplet and radical absorptions, it is very difficult to ascribe physical meaning to each of the (main) components of the distribution. However, it is of interest 
to note that as the PhEA-to-DMNB molar ratio increases, the contribution of the shorter-lived components (tentatively ascribed to DMNB triplets being quenched) tends to increase as well. Thus, amplitude average lifetimes are found to diminish with increasing PhEA concentration (Table 2). While these experimental observations are in full agreement with those previously reported for PhEA/DMNB/ $\beta$-CD solid samples, ${ }^{8,13}$ the data given in Table 2 represent the first set of lifetimes obtained, as a function of varying quencher concentration, for triplet quenching within cyclodextrin solid complexes by means of time-resolved diffuse-reflectance laser-flash photolysis techniques.
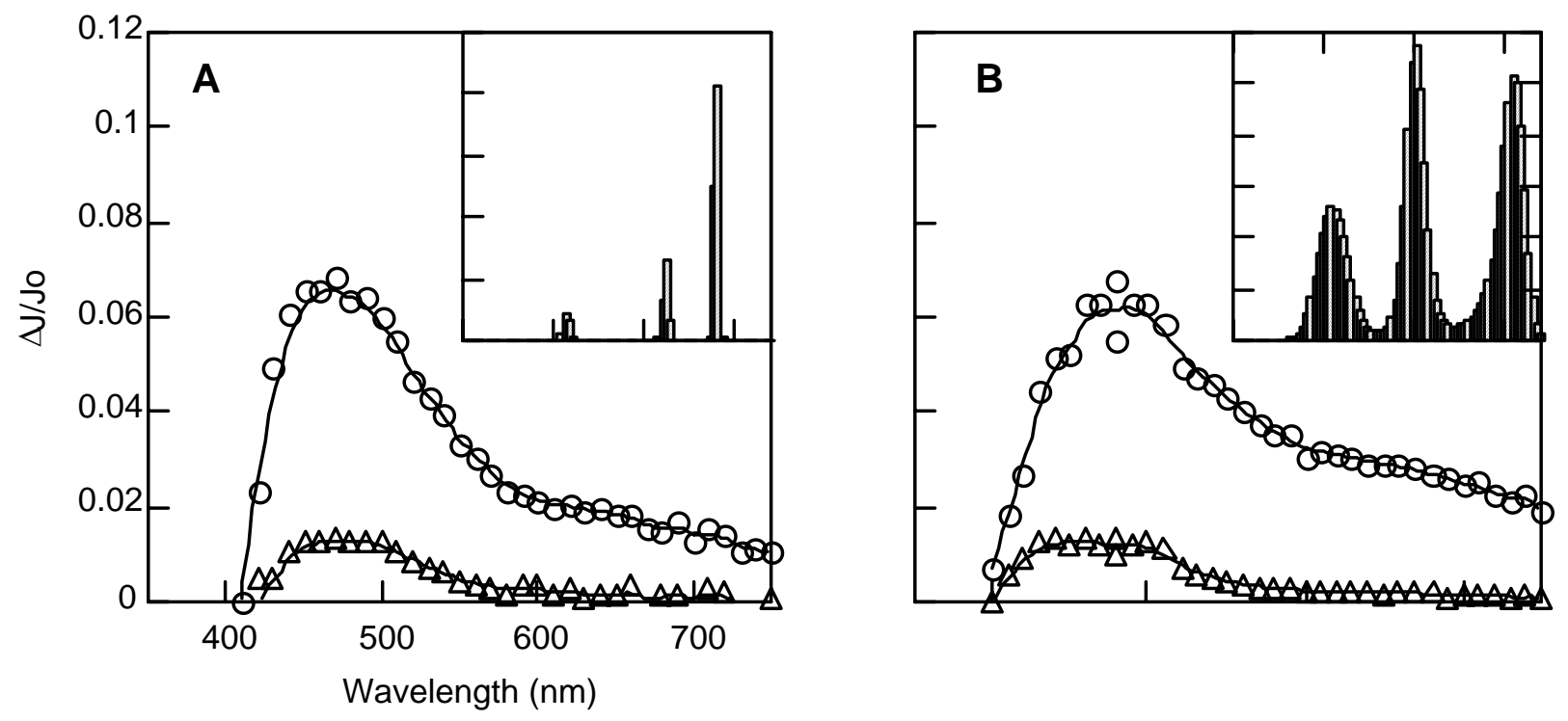

Figure 4. Transient absorption spectra for: (A) DMNB/ $\beta$-CD solid complex (obtained $12.0 \mu \mathrm{s}$ (O) and $142 \mu$ s $(\Delta)$ after laser pulse), and (B) PhEA/DMNB/ $\beta$-CD solid complex (obtained 3.2 $\mu \mathrm{s}(\mathrm{O})$ and $71.2 \mu \mathrm{s}(\Delta)$ after laser pulse). Inset: lifetime distributions ( $\left.\lambda_{\text {monitoring }}=470 \mathrm{~nm}\right)$; molar ratio (A) 0.01:1, (B) 0.07:0.01:1.

Table 2. Amplitude average lifetimes for PhEA/DMNB/ $\beta$-CD solid complexes

\begin{tabular}{cccc}
\hline$[\mathrm{PhEA}]^{\mathrm{b}}$ & $\left\langle\tau>_{\mathrm{a}} / \mu \mathrm{s}\right.$ & {$[\mathrm{PhEA}]^{\mathrm{b}}$} & $\left\langle\tau>_{\mathrm{a}} / \mu \mathrm{s}\right.$ \\
\hline $0^{\mathrm{c}}$ & 55 & 6.5 & 41 \\
0.75 & 51 & 8 & 30 \\
3.5 & 45 & 9 & 25 \\
6 & 39 & 16 & 28 \\
\hline
\end{tabular}

${ }^{\mathrm{a}}$ Concentration of PhEA given in terms of PhEA-to-DMNB molar ratio. ${ }^{\mathrm{b}}$ DMNB-to- $\beta C D$ molar ratio ca. 0.04:1, unless stated otherwise. ${ }^{\mathrm{C}} \mathrm{DMNB}$-to- $\beta$ CD molar ratio 0.01:1. 


\section{Conclusions}

The triplet state dynamics of a series of binary and ternary cyclodextrin solid complexes (air equilibrated samples) have been characterized by means of amplitude average lifetimes, determined by the exponential series method. For comparative purposes, decay traces for binary complexes were also fitted to the Albery model and to either a single or double-exponential function. In the case of binary complexes, resulting host-to-guest molar ratios can easily be correlated with the size/shape of the guest species and the space of the host cavity available for inclusion. Correlation of amplitude average lifetimes with guest molar ratios in ternary cyclodextrin complexes has proven suitable in characterizing solid-state triplet quenching.

\section{Experimental Section}

General Procedures. $\alpha$-Terthienyl (Aldrich, 99\%), $\alpha$-, $\beta$ - and $\gamma$-cyclodextrin, 1,2-dimethoxy-4nitrobenzene, and 1-phenylethylamine (Aldrich), diethyl ether (BDH) and dimethylsulfoxide (Omnisolv) were used as received. 9,10-Phenanthrenequinone, benzil, 1,4-benzoquinone (Aldrich), 9,10-anthraquinone (BDH), and 2,5-dimethyl-1,4-benzoquinone (Eastman) were recrystallized before use. Water was purified by passage through a Millipore Milli-Q system.

With the exception of DMNB complexes, solid complexes were prepared by means of the two-layer precipitation method. ${ }^{29}$ Thus, a concentrated solution of the guest compound in diethyl ether (typically $10 \mathrm{~mL}$ ) was layered over a saturated aqueous cyclodextrin solution (typically 25 $\mathrm{mL}$ ); complexes formed at the interface as a precipitate were then filtered out, washed thoroughly with diethyl ether (to remove any adhered substance) and finally dried in vacuum overnight.

DMNB solid complexes were prepared by means of the cooling method. ${ }^{29}$ Thus, aqueous solutions (typically $5 \mathrm{~mL}$ ) containing $\beta$-CD (ca. $0.4 \mathrm{~g}$ ), DMNB (3-5 mg) and PhEA in varying amounts (i.e., from 4 to $32 \mu \mathrm{L}$ ) were kept (with magnetic stirring) at $70{ }^{\circ} \mathrm{C}$ for 4 hours, and subsequently allowed to cool down slowly to room temperature; complexes formed as a precipitate were then filtered out, washed thoroughly with diethyl ether (to remove any adhered substance) and finally dried in vacuum overnight. Attempts to prepare these complexes by evaporation as described in ref. 8 proved unsuccessful since essentially all DMNB was lost during the washing step with diethyl ether.

Host-to-guest molar ratios were determined by means of UV-visible absorption spectroscopy using $\mathrm{pH} 13$ aqueous buffer (DMNB complexes) or dimethylsulfoxide solutions (all other complexes), as previously described. ${ }^{5}$

Laser experiments were carried out using a Nd/YAG laser (Continuum, Surelite I-10) operated at $355 \mathrm{~nm}$ (4-6 ns pulses, < $15 \mathrm{~mJ} /$ pulse) for excitation. Solid samples, contained in quartz cells constructed of $3 \mathrm{~mm} \times 7 \mathrm{~mm}$ Suprasil tubing, were shaken after every laser pulse to ensure the irradiation of fresh portions of sample by each laser pulse. Further details on the time- 
resolved laser-flash photolysis system employed in this study are reported elsewhere. ${ }^{10}$ All measurements were carried out at $(21 \pm 1)^{\circ} \mathrm{C}$.

Lifetime distribution analyses were carried out by using the exponential series method software (modified version for transient absorption) from Photon Technology International. The fitting function consisted of 100 exponentials terms (with fixed, logarithmically spaced lifetimes) and variable pre-exponential factors. Lifetimes ranged from 10 ns (shortest lifetime allowed) to $250 \mu$ s (limit of our laser flash photolysis system). Alternatively, fittings to the Albery model (using a convenient fitting function as described in ref. 30), and to a single or double exponential function were carried out by using the general curve fitting procedure of Kaleidagraph 3.0.5 software from Abelbeck Software.

\section{Acknowledgements}

Financial support from the Natural Sciences and Engineering Research Council (NSERC) of Canada is gratefully acknowledged.

\section{References}

1. Szejti, J. Cyclodextrins and their Inclusion Complexes; Akadémiai Kiadó: Budapest, 1982.

2. Easton, C. J.; Lincoln, S. L. Modified Cyclodextrins: Scaffolds and Templates for Supramolecular Chemistry; Imperial College Press: London, 1999.

3. Rammamurthy, V., Ed. Photochemistry in Organized and Constrained Media, VCH Publishers: New York, 1991.

4. Koshioka, M.; Mizuma, H.; Imagi, K.; Ikeda, N.; Fukumura, H.; Masuhara, H.; Kryschi, C. Bull. Chem. Soc. Jpn. 1990, 63, 3495.

5. Barra, M.; Bohne, C.; Scaiano, J. C. Photochem. Photobiol. 1991, 54, 1.

6. Barra, M.; Scaiano, J. C. Photochem. Photobiol. 1995, 62, 60.

7. Bourdelande, J. L.; Font, J.; Gonzalez-Moreno, R. J. Photochem. Photobiol. A: Chem. 1996, 94, 215.

8. Mir, M.; Wilkinson, F.; Worrall, D. R.; Bourdelande, J. L.; Marquet, J. J. Photochem. Photobiol. A: Chem. 1997, 111, 241.

9. Milewski, M.; Sikorski, M.; Maciejewski, A.; Mir, M.; Wilkinson, F. J. Chem. Soc., Faraday Trans. 1997, 93, 3029.

10. Barra, M.; Agha, K. A. J. Photochem. Photobiol., A: Chem. 1997, 109, 293.

11. Rao, K. S. S. P.; Hubig, S. M.; Moorthy, J. N.; Kochi, J. K. J. Org. Chem. 1999, 64, 8098.

12. Netto-Ferreira, J. C.; Wintgens, V.; Vieira Ferreira, L. F.; Garcia, A. R.; Ilharco, L. M.; Lemos, M. J. J. Photochem. Photobiol. A: Chem. 2000, 132, 209. 
13. Massot, O.; Mir, M.; Parella, T.; Bourdelande, J. L.; Marquet, J. Phys. Chem. Chem. Phys. 2002, 4, 216.

14. (a) Bruce, J. M. In The Chemistry of the Quinoid Compounds; Patai, S., Ed.; Wiley: New York, 1974; Vol. 1, Ch. 9. (b) Carmichael, I.; Hug, G. L. In Handbook of Organic Photochemistry, Scaiano, J. C., Ed.; CRC Press: Boca Raton, 1989, Vol. I, Ch. 16. (c) Scaiano, J. C.; Redmond, R. W.; Mehta, B.; Arnason, J. T. Photochem. Photobiol. 1990, 52, 655.

15. Ronfard-Haret, J-C.; Bensasson, R. V.; Amouyal, E. J. Chem. Soc., Faraday Trans. 1 1980, 76, 2432.

16. Encinas, M. V.; Scaiano, J. C. J. Am. Chem. Soc. 1979, 101, 7740.

17. Wilkinson, F.; Willsher, C. J.; Casal, L. J.; Johnston, L. J.; Scaiano, J. C. Can. J. Chem. 1986, 64, 539.

18. Evans, C.; Weir, D.; Scaiano, J. C.; Mac Eachern, A.; Arnason, J. T.; Morand, P.; Hollebone, B.; Leitch, L. C.; Philogene, B. J. R. Photochem. Photobiol. 1986, 44, 441.

19. Evans, C. H.; De Feyter, S.; Viaene, L.; van Stam, J.; De Schryver, F. C. J. Phys. Chem. 1996, 100, 2129.

20. Becker, R. S.; Natarajan, L. V. J. Phys. Chem. 1993, 97, 344.

21. Carapellucci, P. A.; Wolf, H. P.; Weiss, K. J. Am. Chem. Soc. 1969, 91, 4635.

22. (a) Hulme, B. E.; Land, E. J.; Phillips, G. O. J. Chem. Soc., Faraday 1 1972, 68, 1992. (b) Hulme, B. E.; Land, E. J.; Phillips, G. O. J. Chem. Soc., Faraday 1 1972, 68, 2003.

23. Aquino, A. M.; Abelt, C. J.; Berger, K. L.; Darragh, C. M.; Kelley, S. E.; Cossette, M. V. J. Am. Chem. Soc. 1990, 112, 5819.

24. Patel, K. B.; Willson, R. L. J. Chem. Soc., Faraday1 1973, 69, 814

25. Mukai, M.; Yamaushi, S.; Hirota, N. J. J. Phys. Chem. 1992, 96, 3305.

26. Quenching by oxygen has been shown not to be important when triplet precursors are included within CD in the solid state (see references 5, 6, 9 and 10).

27. Sillen, A.; Engelborghs, Y. Photochem. Photobiol. 1998, 67, 475.

28. Pang, L.; Withehead, M. A. Supramol. Chem. 1992, 1, 81.

29. Saenger, W. Angew. Chem. Int. Ed. 1980, 19, 344.

30. Albery, W. J.; Barlett, P. N.; Wilde, C. P.; Darwent, J. R. J. Am. Chem. Soc. 1985, 107, 1854. 